\title{
Urokinase plasminogen activator predicts poor prognosis in hepatocellular carcinoma
}

\author{
Fei-Yu Niu", Chuan Jin", Lei Ma, Yan-Xia Shi, Xiao-Shan Li, Peng Jiang, Sha Gao, Jin-Rong Lin*, \\ Ye Song ${ }^{*} \wedge$ \\ Department of Medical Oncology, Affiliated Cancer Hospital and Institute of Guangzhou Medical University, Guangzhou, China \\ Contributions: (I) Conception and design: Y Song; (II) Administrative support: C Jin; (III) Provision of study materials or patients: Y Song; (IV) \\ Collection and assembly of data: FY Niu; (V) Data analysis and interpretation: JR Lin; (VI) Manuscript writing: All authors; (VII) Final approval of \\ manuscript: All authors. \\ \#These authors contributed equally to this work. \\ *These authors contributed equally for the senior authorship. \\ Correspondence to: Ye Song; Jin-Rong Lin. Department of Medical Oncology, Affiliated Cancer Hospital and Institute of Guangzhou Medical \\ University, Guangzhou 510095, China. Email: 565225131@qq.com; ljr035742@163.com.
}

\begin{abstract}
Background: Hepatocellular carcinoma (HCC) is one of the most common malignancies worldwide and its prognosis remains dismal. Hence, it is important to identify the diagnostic and prognostic biomarkers for HCC. Urokinase plasminogen activator (uPA), an extracellular matrix (ECM)-degrading protease, plays a pivotal role in the invasion and metastasis of HCC.

Methods: To confirm the clinical significance of uPA in HCC, we explored uPA expression in HCC in The Cancer Genome Atlas (TCGA) database. The expression level of uPA was further verified by quantitative reverse transcription polymerized chain reaction (qRT-PCR) in 133 pairs of primary HCC samples. A survival analysis was conducted with the Kaplan-Meier method in the HCC samples and TCGA database.

Results: Our results showed that uPA was overexpressed in HCC and was significantly associated with HCC tumor size $(\mathrm{P}=0.015)$, differentiation grade $(\mathrm{P}=0.028)$, and absence of tumor encapsulation $(\mathrm{P}=0.010)$. Patients with high uPA expression levels had a poor outcome $(\mathrm{P}=0.026)$. TCGA database analysis was also consistent with our experimental results.
\end{abstract}

Conclusions: In conclusion, our findings revealed that uPA was overexpressed in HCC and was related to HCC malignant features including tumor size, differentiation grade and absence of tumor encapsulation. High uPA expression had a shorter survival time. It is a potential prognostic biomarker of HCC.

Keywords: Hepatocellular carcinoma (HCC); urokinase plasminogen activator (uPA); prognosis

Submitted May 31, 2021. Accepted for publication Jul 22, 2021.

doi: 10.21037/jgo-21-343

View this article at: https://dx.doi.org/10.21037/jgo-21-343

\section{Introduction}

Hepatocellular carcinoma (HCC) is the fifth most common malignancy and the fourth leading cause of cancer-related deaths worldwide $(1,2)$. The occurrence and development of HCC has been linked to multiple risk factors, such as alcohol abuse, hepatitis B and C virus infections, aflatoxin, nonalcoholic fatty liver disease, and genetic factors (3). Although therapeutic approaches such as surgical interventions and targeted therapy have improved in recent years, the prognosis for HCC patients is still very poor (4). The metastasis and recurrence of HCC is a major cause

\footnotetext{
$\wedge$ ORCID: 0000-0002-9351-7628.
} 
of death (5). Thus, it is important to identify the novel biomarkers of malignant behavior, and in particular the prognostic biomarkers of HCC.

Urokinase plasminogen activator (uPA) is an extracellular proteolytic enzyme, which has a serine protease domain and plays a significant role in tumor metastasis $(6,7)$. By binding to its cell surface receptor uPAR, uPA catalyzes plasminogen to plasmin, initiating metalloproteinase activation which then directly degrades the extracellular matrix (ECM) $(8,9)$. The degradation of ECM contributes to cancer cell invasion and is the first step in tumor progression. In addition, plasminogen activator inhibitors are the main inhibitors of uPA $(10,11)$. Recent findings have suggested that a high expression of uPA is associated with poor prognosis in several cancers, including breast cancer, lung cancer, ovarian cancer, pancreatic cancer, and soft-tissue sarcoma (12-16). A high expression of uPA is reported to promote tumor cell migration and invasion (17-19). However, the majority of published reports are of small or medium size and the correlation between uPA expression and HCC malignant behavior is rarely reported. Therefore, we will test the uPA expression and evaluate the association with malignant features and poor prognosis of HCC in a large patient cohort. The diagnostic and prognostic value of uPA in HCC remain to be clarified.

In the present study, we investigated the uPA expression in HCC and assessed the correlation between uPA and clinical parameters. We explored the prognostic value of uPA in HCC by conducting a survival analysis. We present the following article in accordance with the REMARK reporting checklist (available at https://dx.doi.org/10.21037/ jgo-21-343).

\section{Methods}

\section{Patients and tissue specimens}

A total of 133 paired HCC tumor and paratumor tissues were collected after hepatic resection at Sun Yat-sen University Cancer Center (Guangzhou, China) from 2004 to 2012 and were stored at $-80{ }^{\circ} \mathrm{C}$ immediately. None of the patients had received preoperative treatments, such as chemotherapy or radiotherapy. All patients were histologically diagnosed with primary HCC and chosen randomized. All procedures performed in this study involving human participants were in accordance with the Declaration of Helsinki (as revised in 2013). The study was approved by ethics board of Sun Yat-sen University and informed consent was taken from all the patients.

\section{Quantitative reverse transcription polymerized chain reaction ( $q R T-P C R)$}

TRIzol (Invitrogen, Calsbad, CA, USA) was used to extract total RNA from the clinical tissues. One $\mu \mathrm{g}$ mRNA was reverse transcribed to cDNA with random primers using a PrimeScript ${ }^{\mathrm{RT}}$ reagent kit (Promega, Madison, WI, USA) according to the manufacturer's instructions. qRT-PCR was conducted using the ABI 7900HT Fast Real-Time PCR System (Applied Biosystems, Foster City, CA, USA) with SYBR Green SuperMix (Roche, Basel, Switzerland). The expression level of uPA was normalized against endogenous $\beta$-actin. The primers of uPA were as follows: sense: 5'-GCCATCCCGGACTATACAGA-3', antisence: 5'-AGGCCATTCTCTTCCTTGGT-3'. The primers of $\beta$-actin were as follows: sense: 5 '-GAAGAGCTACGAGCT GCCTGA-3', antisence: 5'-CAGA CAGCACTGTGTT GGCG-3'.

\section{Gene Expression Profiling Interactive Analysis (GEPIA) database analysis}

The GEPIA database is an interactive online cancer web server that facilitates the analysis of RNA sequencing expressions and their prognostic value (http://gepia. cancerpku.cn/index.html). In this study, we analyzed the uPA expression in tumor and nontumor samples in the GEPIA database which were derived from the GenotypeTissue Expression (GTEx) and The Cancer Genome Atlas (TCGA) projects (20). The Student's $t$-test was performed to analyze the differential expression between tumor and nontumor tissues. The cutoff fold-change value was 1 and the cutoff $\mathrm{P}$ value was 0.01 . A survival analysis was also conducted in the GEPIA database. The median was defined as the group cut-off for survival plots.

\section{Statistical analysis}

SPSS standard V.16.0 (SPSS, Inc., IL, USA) was used for the data analysis. Results are presented as means \pm SD for the three independent experiments. The Student's $t$-test was used to compare the differential expression between tumor and nontumor samples. The chi-squared test was used to analyze the association of uPA expression with the clinical-pathological features of HCC patients. The overall 


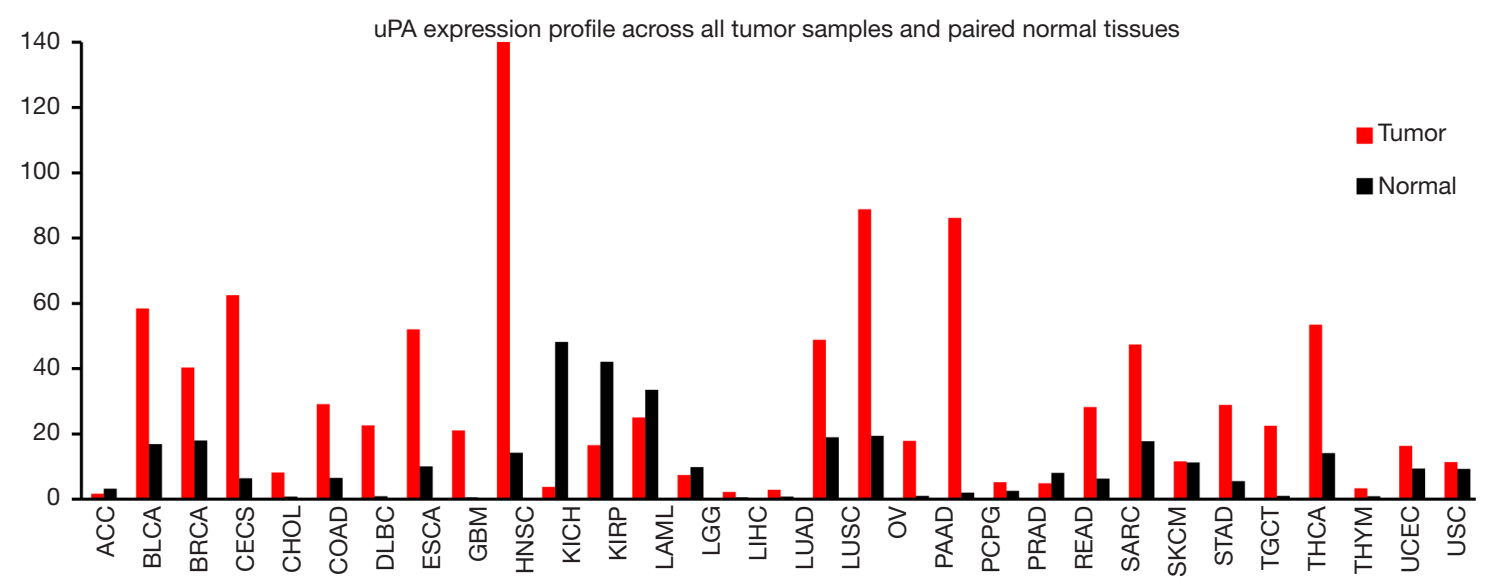

Figure 1 uPA expression levels in different types of cancers. The relative expression levels of uPA in tumor tissues and paired normal tissues in a wide variety of cancers analyzed in the GEPIA database. Data are presented as the mean \pm SD. uPA, urokinase plasminogen activator; GEPIA, Gene Expression Profiling Interactive Analysis.

survival (OS) analysis of HCC patients in the different uPA expression groups was determined by the Kaplan-Meier method, and comparisons were performed with log-rank tests. $P$ values $<0.05$ were considered statistically significant.

\section{Results}

\section{Analysis of uPA overexpression in HCC using the GEPIA database}

We first explored uPA mRNA expression in the GEPIA database. Results showed that uPA was significantly overexpressed in tumor tissues compared with paired normal tissues in many cancer types (Figure 1). We next analyzed uPA expression in HCC and nontumor tissues using TCGA and the GTEx databases. Results demonstrated that uPA was significantly upregulated in 369 HCC tissues compared to 160 nontumor tissues $(\mathrm{P}<0.05$, Figure $2 A)$.

\section{Upregulation of uPA was frequently detected in HCC tissues}

To explore whether uPA expression was higher in HCC tissues than in nontumor tissues, we subsequently tested its expression in 133 pairs of primary HCC samples by qRT-PCR. The results showed that uPA was significantly overexpressed in 51 of the 133 (38.3\%) HCC tissues compared to their nontumor counterparts (defined as $>2$-fold change; Figure 2B). The average expression of uPA in the tumor samples was higher than that in the nontumor samples $(\mathrm{P}<0.05$, paired Student's $t$-test; Figure $2 B)$. Our results demonstrated uPA was frequently overexpressed in HCC tissues.

\section{uPA upregulation was associated with poor prognosis}

To investigate the prognostic value of uPA upregulation in HCC patients, we performed a survival analysis in the GEPIA database and the 133 pairs of primary HCC samples. In the GEPIA database, HCC patients with a high expression of uPA had poorer OS than patients with a low expression of uPA $(\mathrm{P}=0.025$, log-rank test, Figure $3 A)$. We performed a Kaplan-Meier analysis and log-rank tests on the 133 pairs of primary HCC clinical samples. As shown in Figure 3B, the OS rates of HCC patients with high uPA expressions were significantly lower than those with low uPA expressions $(\mathrm{P}=0.026, \log$-rank test). Therefore, overexpression of uPA may be a prognostic factor for HCC.

\section{Clinical significance of uPA in HCC}

To address the clinical value of uPA in HCC, we analyzed the association of uPA overexpression with the clinicalpathological features of 133 HCC patients. The results showed that the overexpression of uPA was significantly associated with HCC tumor size $(\mathrm{P}=0.015)$, differentiation grade $(\mathrm{P}=0.028)$, and absence of tumor encapsulation $(\mathrm{P}=0.010)$ (Table 1), suggesting that uPA may participate in HCC tumor growth and genesis. 

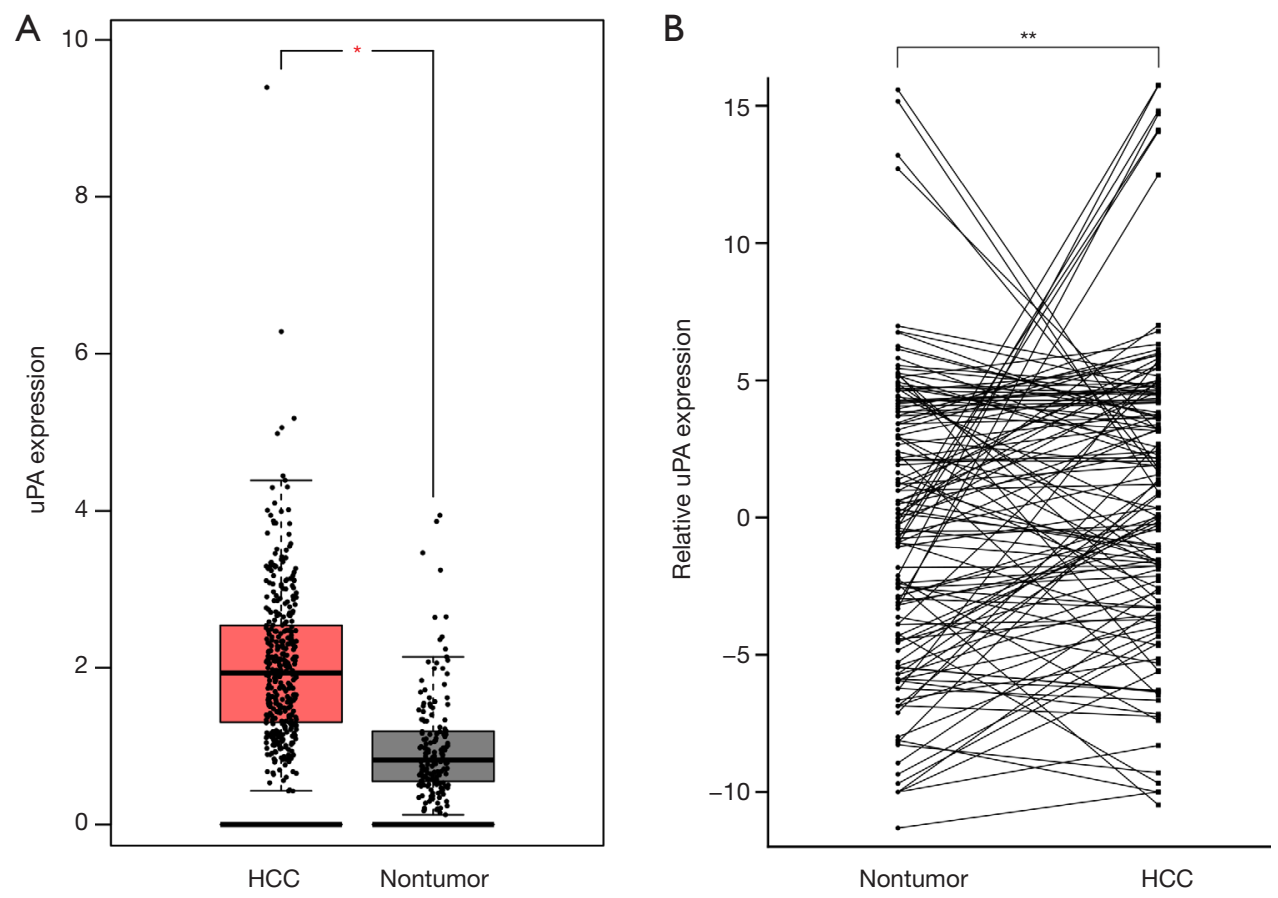

Figure $2 \mathrm{uPA}$ is frequently upregulated in HCC. (A) The bar plot of the uPA expression profile in 369 HCC tissues compared with 160 nontumor tissues. *, $\mathrm{P}<0.01$. (B) The expression of uPA is tested in 133 pairs of primary HCC and their adjacent non-tumor tissues by qRTPCR. Expression of $\beta$-actin is used as an internal control. **, $\mathrm{P}<0.05$, paired Student's $t$-test. uPA, urokinase plasminogen activator; HCC, hepatocellular carcinoma; qRT-PCR, quantitative reverse transcription polymerized chain reaction.
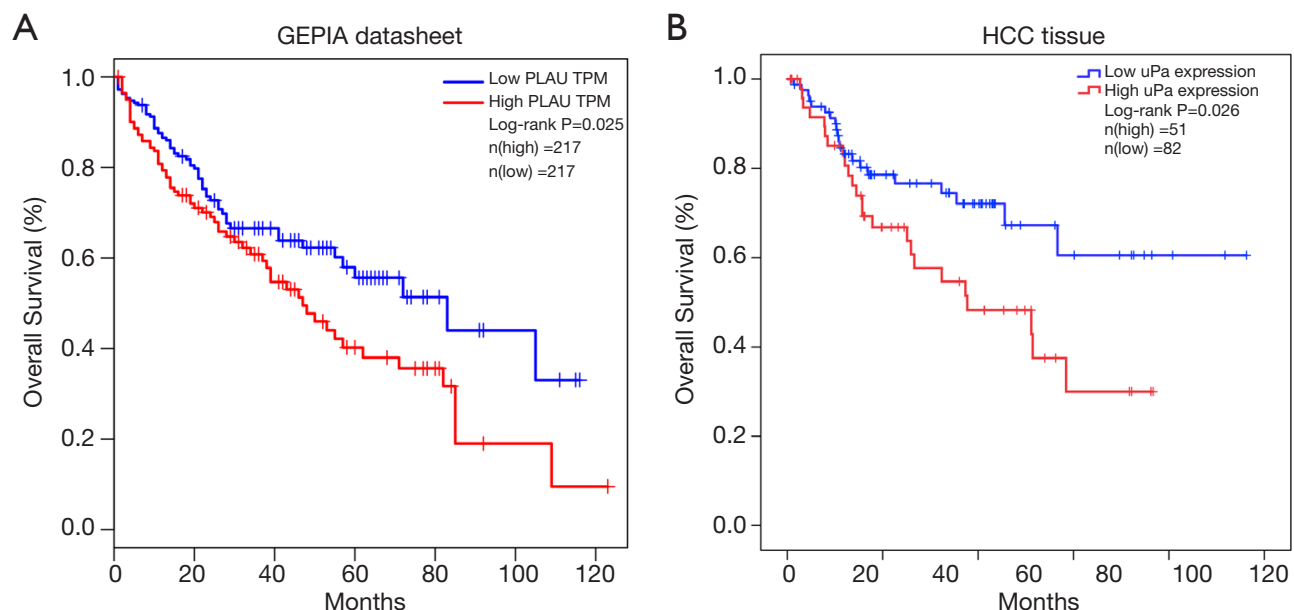

Figure 3 uPA upregulation is associated with poor prognosis. (A) The Kaplan-Meier analysis showing that overexpression of uPA is related to poorer survival of HCC patients in the GEPIA database, P=0.025, log-rank test. (B) The association between uPA expression and OS in HCC patients in the SYSUCC cohort. The Kaplan-Meier test is used to analyze the relationship between OS and uPA expression in patients with HCC. $\mathrm{P}=0.026$, log-rank test. uPA, urokinase plasminogen activator; HCC, hepatocellular carcinoma; GEPIA, Gene Expression Profiling Interactive Analysis; OS, overall survival. 
Table 1 Association of uPA expression with clinicopathological features in HCC

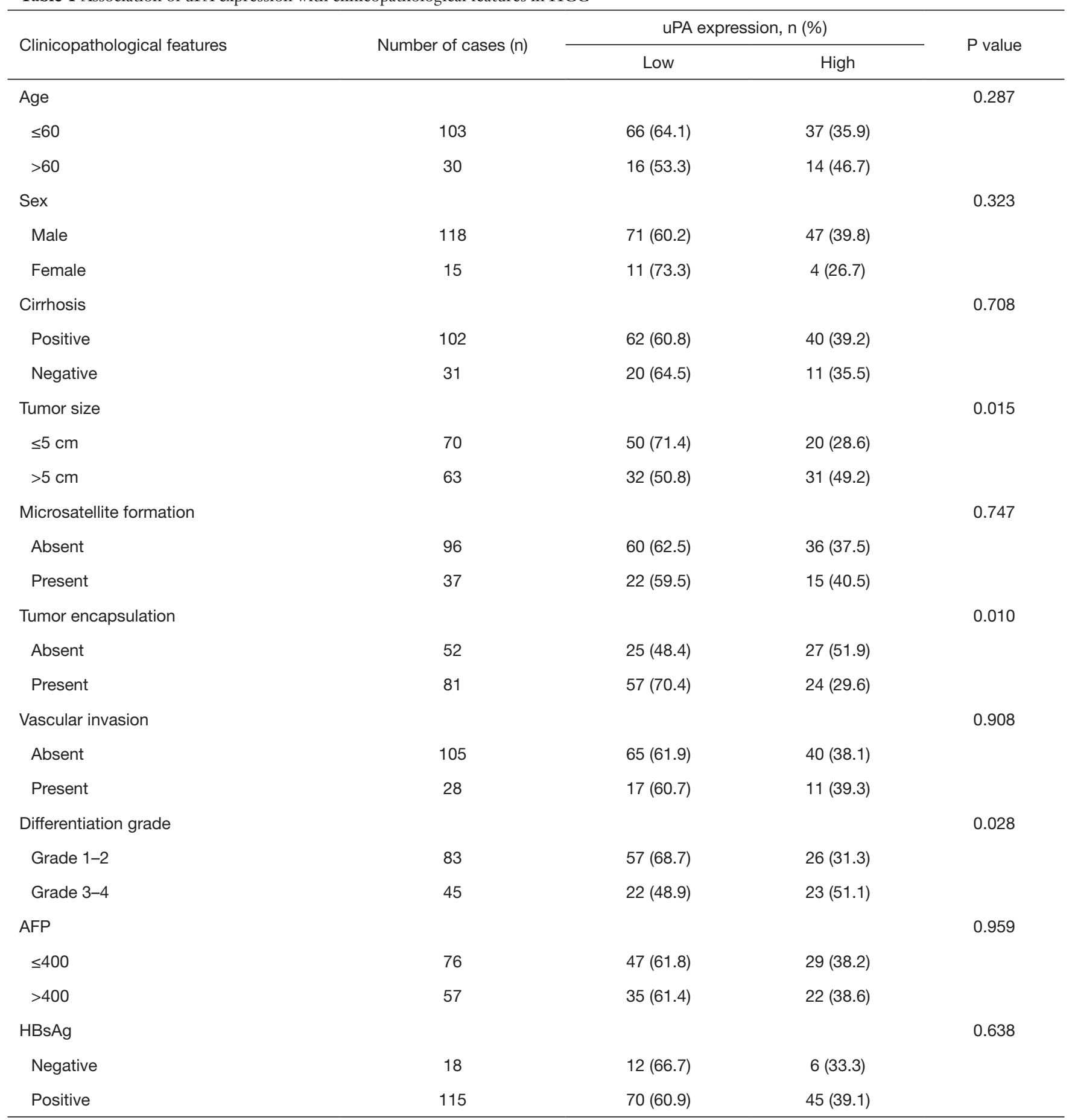

UPA, urokinase plasminogen activator; HCC, hepatocellular carcinoma; AFP, alpha-fetoprotein; HBsAg, hepatitis B virus surface antigen. 


\section{Discussion}

HCC is the fourth leading cause of cancer-related de 3 ths worldwide and accounts for more than $90 \%$ of the histological types of primary liver malignancies (1-3). HCC patients have a high recurrence rate and a poor prognosis (3). Thus, identifying the novel biomarkers of the different pathophysiological stages of HCC is important to improve early disease detection and provide prognostic value for HCC patients.

uPA, a key serine protease, has a restricted substrate specificity to convert the pro-enzyme plasminogen to active serine protease plasmin (21) and is involved in a number of events in the metastatic cascade (22). However, results of in vivo experiments suggest that uPA also cleaves to other proteins in addition to plasminogen, such as alpha6 integrin, fibronectin, uPAR, and hepatocyte growth factor (HGF) (22-24). Plasmin is a broad-spectrum protease and can cleave to multiple substrates including several metalloproteinases which degrade the ECM $(25,26)$, thus promoting cancer cell migration, invasion, and metastasis (27). This process is particularly essential for cancer progression. These reactions are regulated by plasminogen activator inhibitors and by uPA endocytosis $(28,29)$. The plasminogen activator inhibitors PAI-1 and PA1-2 are the main inhibitors of uPA and belong to the serpin family. PAI-1, also known as SERPINE1, is more potent than PAI-2 in inhibiting uPA activity (29-31). PAI-1 balances the adhesion and de-adhesion of cells to the ECM, which is important for tumor cell migration $(32,33)$. Moreover, uPA binding with its receptor uPAR affects migration, adhesion, and metastasis in plasminindependent ways (34). The interaction of uPA with uPAR, subsequently binding with the plasminogen activator inhibitors to form a covalent complex, promotes the uPAuPAR complex to be endocytosed and degraded. Recent evidence has demonstrated that the uPA-uPAR complex interacts with integrins and with several ECM proteins to activate intracellular signaling, such as the RAS/Raf and ERK/MAPK signaling pathways (35-37).

Increasing evidence has identified that the uPA system plays a central role in multiple stages of cancer progression, from tumorigenesis to metastasis (27). Many studies have also demonstrated that elevated expressions in various components of the uPA system are related to poor patient outcomes in a wide variety of cancers (12-16). However, to date there have been few reports concerning uPA expression and function in HCC. Zheng et al. suggest that the overexpression of the uPA system in HCC is associated with
HCC invasiveness, metastasis, and prognosis (38). Other recent research suggests that elevated levels of serum uPA predict poor prognosis in HCC after resection (39).

In order to explore the expression of uPA in HCC and paired nontumor tissues, we identified the uPA mRNA expression in HCC samples by analyzing gene expressions and clinical prognostic data in the GEPIA and TCGA databases and clinical tissues from our hospital. However, the protein level of uPA in HCC is still unknown, Further Western blot and IHC experiments should be performed to investigate protein expression of uPA in HCC tissues in the future. Our analysis found that uPA was consistently overexpressed in many cancers, suggesting that uPA may act as an oncogene in tumorigenesis in a wide variety of cancers. uPA was significantly overexpressed in HCC tissues compared with paired normal tissues, which indicates the significance of uPA in HCC genesis and progression.

In the present study, the expression of uPA in the GEPIA database and 133 pairs of primary HCCs samples were analyzed, and patients with a high expression of uPA had a poorer OS. These data indicated that an elevated uPA level is an OS prognostic factor for HCC patients. Our results are consistent with other research in lung cancer, breast cancer, and pancreatic cancer (12-14). Our study found that the overexpression of uPA was significantly associated with the absence of HCC tumor encapsulation, which provides additional evidence that a high expression of uPA may be involved in the development and progression of HCC. Metalloproteinase activation and ECM degradation are responsible for HCC encapsulation. Overexpression of uPA may convert the pro-enzyme plasminogen to active plasmin thus activating matrix metalloproteinases (MMPs), which subsequently degrade the ECM $(25,26)$. Likewise, in our study, the overexpression of uPA was also associated with HCC tumor size and grade, suggesting that uPA may participate in HCC tumor growth and genesis. It has been reported that uPA can activate several intracellular signaling pathways such as the RAS/Raf and ERK/MAPK pathways to promote tumor growth and metastasis in plasminindependent ways (40).

The metastasis and recurrence of HCC is a major cause of death. The liver is an important organ containing much ECM, the degradation of which is important for tumor genesis and migration, and uPA is known to participate in ECM degradation $(25,26)$. In present study, Higher uPA expression is correlated with absence of tumor encapsulation, suggesting uPA may take part in HCC metastasis through degrade tumor ECM. Further 
mechanism experiments should be performed. uPA expression is also higher in bad differentiation grade and poor outcome patients, indicating that it would serve as a promising prognostic biomarker for HCC.

In the present study, we found that uPA was upregulated in HCC tissues compared with nontumor tissues, and uPA may therefore be a potential prognostic indicator for HCC patients. The association of high uPA expression with the malignant features of HCC indicates that uPA is involved in the development and progression of HCC. However, the potential molecular mechanism remains unknown. Further in vitro and in vivo experiments should be performed to investigate the role of uPA in HCC genesis and progression.

\section{Conclusions}

uPA is overexpressed in HCC and is related to the malignant features and poor prognosis of HCC. Our results suggest that uPA is a potential prognostic biomarker of HCC.

\section{Acknowledgments}

Funding: This work was supported by the National Natural Science Foundation of China (81802335), the National Natural Science Foundation of Guangdong Province (2018A030310175), the Young Doctoral Plan of Guangzhou Medical University (2016C42), the Young Doctoral Plan of Guangdong Health Commission (B2018059), and the General Guidance Plan of Guangzhou Health Commission (20181A010062).

\section{Footnote}

Reporting Checklist: The authors have completed the REMARK reporting checklist. Available at https://dx.doi. org/10.21037/jgo-21-343

Data Sharing Statement: Available at https://dx.doi. org/10.21037/jgo-21-343

Conflicts of Interest: All authors have completed the ICMJE uniform disclosure form (available at https://dx.doi. org/10.21037/jgo-21-343). The authors have no conflicts of interest to declare.

Ethical Statement: The authors are accountable for all aspects of the work in ensuring that questions related to the accuracy or integrity of any part of the work are appropriately investigated and resolved. All procedures performed in this study involving human participants were in accordance with the Declaration of Helsinki (as revised in 2013). The study was approved by ethics board of Sun Yat-sen University and informed consent was taken from all the patients.

Open Access Statement: This is an Open Access article distributed in accordance with the Creative Commons Attribution-NonCommercial-NoDerivs 4.0 International License (CC BY-NC-ND 4.0), which permits the noncommercial replication and distribution of the article with the strict proviso that no changes or edits are made and the original work is properly cited (including links to both the formal publication through the relevant DOI and the license). See: https://creativecommons.org/licenses/by-nc-nd/4.0/.

\section{References}

1. Llovet JM, Zucman-Rossi J, Pikarsky E, et al. Hepatocellular carcinoma. Nat Rev Dis Primers 2016;2:16018.

2. Torre LA, Bray F, Siegel RL, et al. Global cancer statistics, 2012. CA Cancer J Clin 2015;65:87-108.

3. Mittal S, El-Serag HB. Epidemiology of hepatocellular carcinoma: consider the population. J Clin Gastroenterol 2013;47 Suppl:S2-6.

4. Forner A, Llovet JM, Bruix J. Hepatocellular carcinoma. Lancet 2012;379:1245-55.

5. Ilikhan SU, Bilici M, Sahin H, et al. Assessment of the correlation between serum prolidase and alpha-fetoprotein levels in patients with hepatocellular carcinoma. World J Gastroenterol 2015;21:6999-7007.

6. Mekkawy AH, Pourgholami MH, Morris DL. Involvement of urokinase-type plasminogen activator system in cancer: an overview. Med Res Rev 2014;34:918-56.

7. Dass K, Ahmad A, Azmi AS, et al. Evolving role of uPA/uPAR system in human cancers. Cancer Treat Rev 2008;34:122-36.

8. Pillay V, Dass CR, Choong PF. The urokinase plasminogen activator receptor as a gene therapy target for cancer. Trends Biotechnol 2007;25:33-9.

9. Degryse B. The urokinase receptor system as strategic therapeutic target: challenges for the 21 st century. Curr Pharm Des 2011;17:1872-3.

10. Duffy MJ. The urokinase plasminogen activator system: role in malignancy. Curr Pharm Des 2004;10:39-49. 
11. Blasi F, Carmeliet P. uPAR: a versatile signalling orchestrator. Nat Rev Mol Cell Biol 2002;3:932-43.

12. Duffy MJ, Duggan C. The urokinase plasminogen activator system: a rich source of tumour markers for the individualised management of patients with cancer. Clin Biochem 2004;37:541-8.

13. Duffy MJ. Urokinase plasminogen activator and its inhibitor, PAI-1, as prognostic markers in breast cancer: from pilot to level 1 evidence studies. Clin Chem 2002;48:1194-7.

14. Shin SJ, Kim KO, Kim MK, et al. Expression of E-cadherin and $\mathrm{uPA}$ and their association with the prognosis of pancreatic cancer. Jpn J Clin Oncol 2005;35:342-8.

15. Taubert H, Wurl P, Greither T, et al. Co-detection of members of the urokinase plasminogen activator system in tumour tissue and serum correlates with a poor prognosis for soft-tissue sarcoma patients. Br J Cancer 2010;102:731-7.

16. Pappot H, Pedersen AN, Brunner N, et al. The complex between urokinase (uPA) and its type-1 inhibitor (PAI-1) in pulmonary adenocarcinoma: relation to prognosis. Lung Cancer 2006;51:193-200.

17. Shi Z, Stack MS. Urinary-type plasminogen activator (uPA) and its receptor (uPAR) in squamous cell carcinoma of the oral cavity. Biochem J 2007;407:153-9.

18. Li HF, Liu YQ, Shen ZJ, et al. Downregulation of MACC1 inhibits invasion, migration and proliferation, attenuates cisplatin resistance and induces apoptosis in tongue squamous cell carcinoma. Oncol Rep 2015;33:651-60.

19. Nozaki S, Endo Y, Kawashiri S, et al. Immunohistochemical localization of a urokinase-type plasminogen activator system in squamous cell carcinoma of the oral cavity: association with mode of invasion and lymph node metastasis. Oral Oncol 1998;34:58-62.

20. Tang Z, Li C, Kang B, et al. GEPIA: a web server for cancer and normal gene expression profiling and interactive analyses. Nucleic Acids Res 2017;45:W98-102.

21. Andreasen PA, Egelund R, Petersen HH. The plasminogen activation system in tumor growth, invasion, and metastasis. Cell Mol Life Sci 2000;57:25-40.

22. Andreasen PA, Kjoller L, Christensen L, et al. The urokinase-type plasminogen activator system in cancer metastasis: a review. Int J Cancer 1997;72:1-22.

23. Binder BR, Mihaly J, Prager GW. uPAR-uPA-PAI-1 interactions and signaling: a vascular biologist's view. Thromb Haemost 2007;97:336-42.

24. Naldini L, Tamagnone L, Vigna E, et al. Extracellular proteolytic cleavage by urokinase is required for activation of hepatocyte growth factor/scatter factor. EMBO J 1992;11:4825-33.

25. Duffy MJ. The role of proteolytic enzymes in cancer invasion and metastasis. Clin Exp Metastasis 1992;10:145-55.

26. Su SC, Lin CW, Yang WE, et al. The urokinase-type plasminogen activator (uPA) system as a biomarker and therapeutic target in human malignancies. Expert Opin Ther Targets 2016;20:551-66.

27. Choong PF, Nadesapillai AP. Urokinase plasminogen activator system: a multifunctional role in tumor progression and metastasis. Clin Orthop Relat Res 2003;(415 Suppl):S46-58.

28. Andreasen PA, Georg B, Lund LR, et al. Plasminogen activator inhibitors: hormonally regulated serpins. Mol Cell Endocrinol 1990;68:1-19.

29. Halamkova J, Kiss I, Pavlovsky Z, et al. Clinical relevance of uPA, uPAR, PAI 1 and PAI 2 tissue expression and plasma PAI 1 level in colorectal carcinoma patients. Hepatogastroenterology 2011;58:1918-25.

30. Wang L, Ly CM, Ko CY, et al. uPA binding to PAI1 induces corneal myofibroblast differentiation on vitronectin. Invest Ophthalmol Vis Sci 2012;53:4765-75.

31. Harbeck N, Schmitt M, Meisner C, et al. Ten-year analysis of the prospective multicentre Chemo-N0 trial validates American Society of Clinical Oncology (ASCO)recommended biomarkers uPA and PAI-1 for therapy decision making in node-negative breast cancer patients. Eur J Cancer 2013;49:1825-35.

32. Simone TM, Longmate WM, Law BK, et al. Targeted inhibition of PAI-1 activity impairs epithelial migration and wound closure following cutaneous injury. Adv Wound Care (New Rochelle) 2015;4:321-8.

33. Van De Craen B, Declerck PJ, Gils A. The biochemistry, physiology and pathological roles of PAI-1 and the requirements for PAI-1 inhibition in vivo. Thromb Res 2012;130:576-85.

34. Danø K, Andreasen PA, Grøndahl-Hansen J, et al. Plasminogen activators, tissue degradation, and cancer. Adv Cancer Res 1985;44:139-266.

35. Tang L, Han X. The urokinase plasminogen activator system in breast cancer invasion and metastasis. Biomed Pharmacother 2013;67:179-82.

36. Chandrasekar N, Mohanam S, Gujrati M, et al. Downregulation of uPA inhibits migration and PI3k/Akt signaling in glioblastoma cells. Oncogene $2003 ; 22: 392-400$. 
37. Ghosh S, Koblinski J, Johnson J, et al. Urinary-type plasminogen activator receptor/alpha 3 beta 1 integrin signaling, altered gene expression, and oral tumor progression. Mol Cancer Res 2010;8:145-58.

38. Zheng Q, Tang ZY, Xue Q, et al. Invasion and metastasis of hepatocellular carcinoma in relation to urokinase-type plasminogen activator, its receptor and inhibitor. J Cancer Res Clin Oncol 2000;126:641-6.

39. Tsai MC, Yen YH, Chang KC, et al. Elevated levels of

Cite this article as: Niu FY, Jin C, Ma L, Shi YX, Li XS, Jiang P, Gao S, Lin JR, Song Y. Urokinase plasminogen activator predicts poor prognosis in hepatocellular carcinoma. J Gastrointest Oncol 2021;12(4):1851-1859. doi: 10.21037/jgo21-343 serum urokinase plasminogen activator predict poor prognosis in hepatocellular carcinoma after resection. BMC Cancer 2019;19:1169.

40. Luo J, Sun X, Gao F, et al. Effects of ulinastatin and docetaxel on breast cancer invasion and expression of uPA, uPAR and ERK. J Exp Clin Cancer Res 2011;30:71.

(English Language Editor: D. Fitzgerald) 